\title{
The dielectric signature of poly( $N$-isopropylacrylamide) microgels at the volume phase transition: dependence on the crosslinking density
}

\begin{abstract}
Marieke Füllbrandt, ${ }^{\text {ab }}$ Regine von Klitzing ${ }^{b}$ and Andreas Schönhals ${ }^{* a}$
Temperature sensitive poly( $N$-isopropylacrylamide) (pNIPAM) microgels are prepared and investigated using dielectric spectroscopy in a frequency range from $10^{-1} \mathrm{~Hz}$ to $10^{6} \mathrm{~Hz}$ at temperatures from $15^{\circ} \mathrm{C}$ to $50{ }^{\circ} \mathrm{C}$. The microgels were synthesized with different crosslinker molar ratios resulting in microgels with structural differences. From the dielectric response of the pNIPAM microgels the swelling/ deswelling behaviour is monitored by both the temperature $(T)$ and the frequency $(f)$ dependence of the conductivity spectra $\sigma^{*}(f, T)$. The volume phase transition (VPT) at the lower critical solution temperature (LCST) is deduced by a change in the $T$-dependence of the DC conductivity $\sigma_{\mathrm{DC}}^{\prime}$. It can be explained by a decrease in the effective charge mobility and a reduction in the effective charge number contributing to $\sigma_{\mathrm{DC}}^{\prime}$ at $T>\mathrm{LCST}$. Addressing the $f$-dependence of the real part of the conductivity $\sigma^{\prime}$, a pronounced frequency dependence at temperatures above the LCST can be observed whereas at temperatures below the LCST the conductivity spectra resemble that of the pure solvent (water) which is frequency independent. The $f$-dependence of $\sigma^{\prime}$ at $T>$ LCST is assigned to the collapse of the microgel particles. At the interfaces of the collapsed particles charge carriers are blocked and/or entrapped giving rise to Maxwell-Wagner-Sillars (MWS) polarization effects. The dependence of the MWS effect on the crosslinker amount is studied in detail and conclusions concerning the internal structure of the microgels with respect to their crosslinking density are drawn. Moreover the dielectric data are related to dynamic light scattering data. A correlation between the MWS polarization effect and the swelling/ deswelling ratio expressed by the hydrodynamic radius $R_{\mathrm{h}}$ at different temperatures is established for the first time.
\end{abstract}

Received 30th November 2012

Accepted 5th March 2013

DOI: $10.1039 / \mathrm{c} 3 \mathrm{sm} 27762 \mathrm{c}$

www.rsc.org/softmatter also called volume phase transition temperature (VPTT). ${ }^{5}$ The volume phase transition (VPT) is a reversible process. The macroscopic driving force for the volume change (collapse) is related to the competition between mainly two molecular effects, hydrogen bonding and hydrophobic interaction. ${ }^{9}$

Microgels composed of pNIPAM were first synthesized by Pelton and Chibante in 1986 using a precipitation polymerisation (emulsion polymerisation). ${ }^{\mathbf{1 0}}$ Monodisperse microgel particles can be obtained, however the general procedure of microgel synthesis leads to an inhomogeneous distribution with a higher crosslinker density in the particle core and a higher charge density in the particle shell. ${ }^{7,11}$ This results in particles with a core-shell structure with a harder inner core and a softer shell being a more porous and permeable layer consisting of dangling or lightly crosslinked chains leading to a quite complex shrinking behaviour. Recently, Varga et al. suggested a new type of semi-batch reactor, which allows the synthesis of microgel particles with a more uniform crosslinker density. ${ }^{12}$ The amount and distribution of the crosslinker just as the charge density strongly affect the microgel properties, e.g. the internal mobility and its mechanical characteristics. ${ }^{13}$ 
The internal structure as well as thermodynamic and kinetic aspects of the VPT of pNIPAM microgels have been studied by various methods, including dynamic light scattering, ${ }^{\mathbf{8 , 1 4}, 15}$ microscopy, ${ }^{14,16}$ small angle neutron scattering, ${ }^{14,17,18}$ and neutron spin echo spectroscopy. ${ }^{19}$ Structural heterogeneity has been also investigated using dye labelling microgels ${ }^{\mathbf{2 0 2 1}}$ or by incorporation of nanoparticles (e.g. Au).$^{22}$ However, for investigations of the dynamics visual techniques are in general not useful. A theoretical model based on the terminal copolymerization kinetics which predicts the microstructure of functionalized microgels was developed by Hoare and McLean. ${ }^{23,24}$ Monomer and functional group distribution in multicomponent pNIPAM based microgels can be predicted by linking it to the copolymerization kinetics of the respective comonomers. The control over microgel morphology and its proper characterization is a key area of research.

The present study introduces a new approach for the investigation of the induced responsive behaviour of microgels in the bulk phase and the related internal structure. Dielectric spectroscopy is applied to pNIPAM microgels in aqueous media. It is a well suited, non-invasive technique to study the structure and dynamics of polymers including their solutions. Dielectric spectroscopy covers a broad frequency range, thus processes on different time and length scales can be studied by taking the molecular mobility and/or the conductivity as a probe for the structure. ${ }^{25}$ There are only a few publications on the dielectric behaviour of pNIPAM in solution. Ono and Shikata ${ }^{26,27}$ studied the hydration and molecular dynamics at temperatures between $6{ }^{\circ} \mathrm{C}$ and $39^{\circ} \mathrm{C}$ of linear pNIPAM and Nakano et al. investigated the relaxation processes of linear pNIPAM and solvent molecules as a function of polymer concentration and type of solvent. ${ }^{28}$ Dielectric investigations on pNIPAM microgels were recently published by Gómez-Galván et $a .^{29}$ and Zhou et al. ${ }^{30}$ The former presents a new device for simultaneous measurements of optical and dielectric properties for linear pNIPAM and pNIPAM hydrogels in aqueous solutions. The light transmittance, relative permittivity and the dielectric loss tangent as a function of temperature were measured in the lower frequency region $\left(10^{2} \mathrm{~Hz}\right.$ to $\left.10^{6} \mathrm{~Hz}\right)$ in order to analyse the water-polymer interaction. The latter publication covers about the same frequency range $\left(10^{-2} \mathrm{~Hz}\right.$ to $\left.10^{7} \mathrm{~Hz}\right)$ and connects dielectric and electric properties of structurally different pNIPAM microgels with the gel network. By varying the preparation mechanism pNIPAM microgels with different charge distributions and structures are prepared. In the dielectric spectra the authors detected two relaxation modes, a slow mode ( $\alpha$-dispersion) and a fast mode ( $\delta$-relaxation). Although some of their arguments should be discussed in greater detail the latter was used to differentiate different structures of microgels.

In a recent dielectric study on linear pNIPAM in water ${ }^{31}$ we proved that the phase transition behaviour can be monitored by both the temperature $(T)$ and frequency $(f)$ dependence of the complex conductivity $\sigma^{*}(f, T)$. In the present contribution, pNIPAM microgels with different crosslinking densities are analysed at different temperatures in a frequency range from $10^{-1} \mathrm{~Hz}$ to $10^{6} \mathrm{~Hz}$. The same approach as in ref. 31 is used, which is to take charges as a probe to investigate the internal structure of the microgels. No extra charge carriers have been added to the samples, only remaining charges coming from the polymerization process (e.g. from the initiator) and residual charge carriers in the Milli- $\mathrm{Q}^{\mathrm{TM}}$ water used as a solvent have been considered.

\section{Experimental}

\section{Microgel synthesis}

$N$-Isopropylacrylamide (NIPAM) with a purity of at least $97 \%$, $N, N^{\prime}$-methylenebisacrylamide (BIS) as a crosslinker and potassium persulphate (KPS) as an initiator were purchased from Sigma Aldrich and used without further purification. Poly $(N-$ isopropylacrylamide) (pNIPAM) microgels with different amounts of crosslinker ranging from $0.5 \mathrm{~mol} \%$ to $10 \mathrm{~mol} \%$ with respect to the molar concentration of NIPAM monomers were synthesized by a radical surfactant free emulsion polymerization (for a detailed description see ref. 10). The preparation procedure was as follows: $0.56 \mathrm{~g}$ of monomer (NIPAM) with the respective amount of crosslinker (BIS) was dissolved in Milli$\mathrm{Q}^{\mathrm{TM}}$ water (Millipore, USA), placed under a nitrogen atmosphere and heated up to $70{ }^{\circ} \mathrm{C}$. $10 \mathrm{mg}$ of initiator (KPS) dissolved in Milli- $\mathrm{Q}^{\mathrm{TM}}$ water was added to the mixture to start the reaction. It proceeded for about $4 \mathrm{~h}$ to $5 \mathrm{~h}$. After that time the reaction mixture was cooled down to room temperature overnight. All samples were purified by centrifugation and redispersion in water several times and subsequently the microgels were freezedried. The diameter and the internal structure (mesh pore sizes, crosslinking distribution) of the resulting microgel particles depend on the amount of crosslinker used. All pNIPAM microgels have a low concentration of covalently bound charged groups coming from the persulphate based radical initiator. In fact, zeta potential measurements (results not shown here) elucidated that the synthesized pNIPAM microgels possess a negatively charged surface which can be traced back to the sulphate $\left(\mathrm{SO}_{4}{ }^{-}\right)$groups. The zeta potential calculated from the electrophoretic mobility becomes even more negative as the temperature is increased above the volume phase transition temperature. The higher crosslinked core has a lower volume charge density than the less crosslinked shell. The electrophoretic mobility of the particles is dominated by the charge density within the shell. Upon shell collapsing the number density of charged groups in the surface charge layer increases and hence the mobility increases. ${ }^{32}$

In Fig. 1 some AFM images of the synthesized pNIPAM microgels are presented in a dry and adsorbed state. AFM imaging was performed with a Cypher ${ }^{\mathrm{TM}}$ AFM (Asylum Research, USA) in air and tapping mode at room temperature. For the investigation of individual microgel particles, samples were spin coated on a silica wafer (3000 rpm for $300 \mathrm{~s}$ ) under ambient conditions. The AFM images show the microgel particles to be spherical and monodisperse.

\section{Methods}

Dynamic light scattering (DLS). For a first characterization of the swelling/deswelling behaviour of the microgel particles DLS 

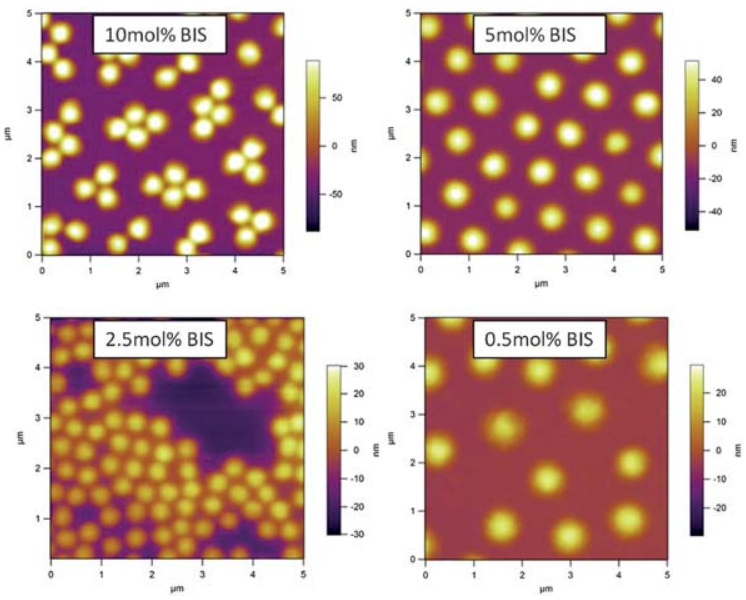

Fig. 1 AFM images for dried microgel particles with different amounts of crosslinker (as indicated) adsorbed on a silica wafer with a native $\mathrm{SiO}_{x}$ layer. Imaging was performed in air and tapping mode at room temperature. Please note the different $z$-scale bars.

measurements were conducted using an ALC/CGS-3 compact goniometer system equipped with an ALV/LSE-5004 correlator. The laser wavelength was $632.8 \mathrm{~nm}$. A temperature range between $15{ }^{\circ} \mathrm{C}$ and $50{ }^{\circ} \mathrm{C}$ was covered where the temperature was controlled by a Huber Compatible Control thermostat with a temperature stability of $0.1 \mathrm{~K}$. Intensity time autocorrelation functions were recorded at a constant scattering angle of $60^{\circ}$ (scattering vector $q=0.013226 \mathrm{~nm}^{-1}$ ). The data are acquired in the form of the reduced intensity autocorrelation function $g^{2}(q, t)$ which is converted by the instruments software to yield the electric field autocorrelation function $g^{1}(q, t)$ via the Siegert relation. ${ }^{33}$ The latter describes the correlated particle movement (fluctuations). For polydisperse systems the autocorrelation function can be described by a sum or distribution of exponential decays:

$$
g^{1}(\tau)=\int G(\Gamma) \exp (-\Gamma \tau) \mathrm{d} \Gamma
$$

where $\Gamma$ is the decay rate which is related to the diffusion coefficient $D$ by $\Gamma=D q^{2}$ and $G(T)$ is the distribution function of the decay rates. The hydrodynamic radius $R_{\mathrm{h}}$ is accessible via the Stokes Einstein equation:

$$
R_{\mathrm{h}}=\frac{k_{\mathrm{B}} T}{6 \pi \eta D}
$$

where $k_{\mathrm{B}}$ is the Boltzmann constant, $T$ is Temperature in units of $\mathrm{K}, \eta$ is the viscosity of the solvent and $D$ the diffusion coefficient. $R_{\mathrm{h}}$ was averaged over five single measurements at each temperature with a standard deviation of $\pm 1 \%$. The concentration of the microgel dispersions for light scattering experiments was $c=10^{-5} \mathrm{~g} \mathrm{ml}^{-1}$. In the present case, the data were analysed using a method based on a regularized inverse Laplace transformation similar to the CONTIN algorithm. ${ }^{34}$ The polydispersity index (PDI) is defined as the second cumulant of the scattered light correlation function analysed using the cumulant method divided by the average decay rate. A fully monodisperse sample would have a PDI of $0 .^{35}$ The obtained values
Table 1 Characteristics of the synthesized microgels as measured by dynamic light scattering: hydrodynamic radius $R_{\mathrm{h}}$ at $20^{\circ} \mathrm{C}$ and $45^{\circ} \mathrm{C}, \Delta R_{\mathrm{h}}\left(=R_{\mathrm{h}, 20^{\circ} \mathrm{C}-}\right.$

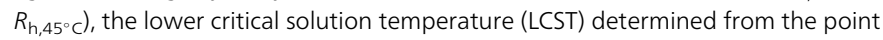
of deflection and the polydispersity index (PDI)

\begin{tabular}{llllll}
\hline $\begin{array}{l}\text { Crosslinker content } \\
{[\mathrm{mol} \%]}\end{array}$ & $\begin{array}{l}R_{\mathrm{h}, 20^{\circ} \mathrm{C}} \\
{[\mathrm{nm}]}\end{array}$ & \begin{tabular}{l}
$R_{\mathrm{h}, 45^{\circ} \mathrm{C}}[\mathrm{nm}]$ \\
\hline 10
\end{tabular} & $\begin{array}{l}\Delta R_{\mathrm{h}} \\
{[\mathrm{nm}]}\end{array}$ & $\begin{array}{l}\text { LCST } \\
{\left[{ }^{\circ} \mathrm{C}\right]}\end{array}$ & $\begin{array}{l}\text { PDI } 20{ }^{\circ} \mathrm{C} / \\
45{ }^{\circ} \mathrm{C}\end{array}$ \\
\hline 5 & 189 & 108 & 81 & 32.5 & $0.31 / 0.26$ \\
2.5 & 286 & 152 & 134 & 32.5 & $0.06 / 0.03$ \\
1 & 293 & 118 & 175 & 33.5 & $0.21 / 0.15$ \\
0.5 & 308 & 120 & 188 & 31.5 & $0.08 / 0.02$ \\
\hline
\end{tabular}

(see Table 1) are sufficiently low indicating a more or less narrow size distribution of the particles in agreement with the AFM results.

Dielectric spectroscopy. The complex dielectric function $\varepsilon^{*}$ is derived by measuring the complex impedance $Z^{*}(\omega)$ of the sample:

$$
\varepsilon^{*}(\omega)=\varepsilon^{\prime}(\omega)-i \varepsilon^{\prime \prime}(\omega)=\frac{1}{i \omega Z^{*}(\omega) C_{0}}
$$

where $\varepsilon^{\prime}$ is the real part, $\varepsilon^{\prime \prime}$ the loss part, $\omega$ the angular frequency $(f=2 \pi \omega), i=(-1)^{1 / 2}$ and $C_{0}$ the capacitance of the empty sample capacitor. $Z^{*}(\omega)$ is measured using Fourier Correlation Analysis. ${ }^{25}$ Measurements were carried out in a frequency range from $10^{-1}$ $\mathrm{Hz}$ to $10^{6} \mathrm{~Hz}$ and between $15^{\circ} \mathrm{C}$ and $50^{\circ} \mathrm{C}$ with a high resolution ALPHA analyser (Novocontrol, Hundsangen, Germany). The temperature is controlled by a Quatro Novocontrol cryo system with a temperature stability of $0.1 \mathrm{~K}$. Microgel dispersions with a concentration of $2.5 \times 10^{-2} \mathrm{~g} \mathrm{ml}^{-1}$ were investigated using a liquid sample cell supplied by Novocontrol (BDS 1309) with a diameter of $11 \mathrm{~mm}$. The cell is made up of two gold plated electrodes with a Teflon cylinder as a spacer in between. The electrode spacing of $5.5 \mathrm{~mm}$ is relatively wide in order to shift the effect of electrode polarization to lower frequencies.

\section{Results and discussion}

The results and discussion part of the paper is organized as follows. First, the light scattering results are shown for diluted microgel dispersions and the swelling/deswelling behaviour in dependence on the crosslinking density is discussed. Second, the dielectric response of pNIPAM microgels is discussed by analysing the temperature and frequency dependence of the complex conductivity. Finally, light scattering and dielectric data are correlated.

\section{Swelling/deswelling behaviour of pNIPAM microgels measured by DLS}

The temperature dependence of the hydrodynamic radius $R_{\mathrm{h}}$ for pNIPAM microgels with different amounts of crosslinker is shown in Fig. 2. All microgels demonstrate the typical thermosensitive behaviour that $R_{\mathrm{h}}$ is decreasing with increasing temperature reaching a plateau at about $40{ }^{\circ} \mathrm{C}$ (collapsed temperature independent state). Moreover, it can be seen that 


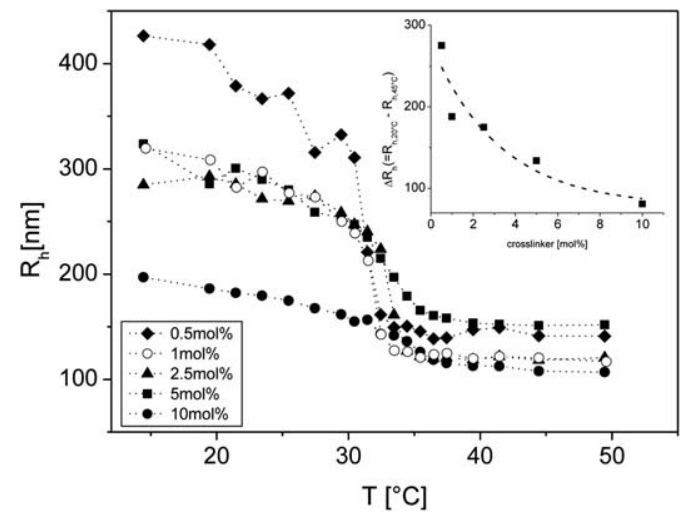

Fig. 2 Temperature dependence of the hydrodynamic radius $R_{\mathrm{h}}$ for pNIPAM microgels with different amounts of crosslinker obtained by dynamic light scattering. The dotted lines are guides to the eyes. The concentration of the microgel dispersions is $10^{-5} \mathrm{~g} \mathrm{ml}^{-1}$. The inset shows the dependence of $\Delta R_{\mathrm{h}}\left(=R_{\mathrm{h}, 20^{\circ} \mathrm{C}}-\right.$ $R_{\left.\mathrm{h}, 45^{\circ} \mathrm{C}\right)}$ on the crosslinker amount. The dashed line is an exponential fit to the data (only a guide for the eyes).

with increasing amount of crosslinker, the ability to swell and deswell (here expressed by $\Delta R_{\mathrm{h}}=R_{\mathrm{h}, 20^{\circ} \mathrm{C}}-R_{\mathrm{h}, 45^{\circ} \mathrm{C}}$ ) decreases. This is demonstrated in the inset of Fig. 2 by plotting $\Delta R_{\mathrm{h}}$ versus the concentration of the crosslinker. It is well known that the swelling/deswelling ratio of microgels becomes smaller for higher crosslinked microgels. ${ }^{36,37}$ The absolute size of the microgel particles in the swollen state $(T<$ LCST) depends on the amount of crosslinker and is smaller for gels with higher crosslinker content. However, it is worth noting that the size of the collapsed microgel particle $(T>$ LCST) does not show a systematic dependence on the crosslinker density and varies only little with crosslinking density compared to the particle sizes in the swollen state. The values of the hydrodynamic radii at $20{ }^{\circ} \mathrm{C}\left(R_{\mathrm{h}, 20^{\circ} \mathrm{C}}\right)$ and $45{ }^{\circ} \mathrm{C}\left(R_{\mathrm{h}, 45^{\circ} \mathrm{C}}\right), \Delta R_{\mathrm{h}}$, the lower critical solution temperature (LCST) as well as the polydispersity (PDI) for the different microgels are listed in Table 1. The LCST is determined from the point of deflection of the DLS curves and is basically independent of crosslinker density.

\section{Dielectric response of pNIPAM microgels}

In the considered temperature range $\left(15{ }^{\circ} \mathrm{C}\right.$ to $\left.50{ }^{\circ} \mathrm{C}\right)$ the molecular rotational fluctuations of the water molecules and the segmental mobility of pNIPAM take place at much shorter time scales (i.e. higher frequencies). ${ }^{27,28}$ They cannot be observed in the selected frequency range as characteristic relaxation processes. However, as shown in our recent paper, ${ }^{31}$ interfacial polarization effects such as Maxwell-Wagner-Sillars (MWS) polarization can be detected. Therefore the data are expressed as complex conductivity $\sigma^{*}(\omega)$ since the focus is on the mobility of charges in order to monitor structural changes. $\sigma^{*}(\omega)$ is given by:

$$
\sigma^{*}(\omega)=\sigma^{\prime}(\omega)+i \sigma^{\prime \prime}(\omega)=i \omega \varepsilon_{0} \varepsilon^{*}(\omega)
$$

where $\sigma^{\prime}$ and $\sigma^{\prime \prime}$ are the corresponding real and loss parts, respectively ( $\varepsilon_{0}$ is the permittivity of free space). For details see ref. 25.
The complex conductivity for water in the considered temperature and frequency range is discussed in ref. 31. Besides small electrode polarization effects at low frequencies $\sigma^{\prime}$ is independent of frequency and increases monotonously with temperature. As an example Fig. 3 depicts the frequency $f$ and temperature $T$ dependence of the real part $\sigma^{\prime}$ of complex conductivity for a pNIPAM microgel with $0.5 \mathrm{~mol} \%$ BIS dispersed in water. In contrast to pure water the conductivity spectrum of the pNIPAM microgel shows a pronounced frequency as well as temperature dependence. These dependencies are related to both the drift motion of charge carriers (DC conductivity) and to interfacial polarization effects due to the collapsing of the microgel at the lower critical solution temperature (LCST). Both dependencies will be discussed in more detail in the following.

\section{Temperature dependence of the DC conductivity $\sigma_{\mathrm{DC}}^{\prime}$}

For aqueous systems where no interfacial polarization effects take place the conductivity $\sigma^{\prime}$ is independent of frequency, i.e. it is constant over the whole frequency range and represents the DC conductivity $\left(\sigma^{\prime}\right.$ at $\left.f=0 \mathrm{~Hz}\right)$. However, due to interfacial polarization effects (electrode and/or MWS polarization) a frequency dependence can be observed for $\sigma^{\prime}$. Therefore the DC conductivity has to be defined in a region where these effects can be excluded and $\sigma^{\prime} v$ s. frequency shows a constant value. As discussed in ref. 31 the conductivity $\sigma^{\prime}$ shows a plateau region at high frequencies corresponding to the DC conductivity of the system $\left(\sigma_{\mathrm{DC}}^{\prime}\right)$. For a better understanding, the frequency dependence of $\sigma^{\prime}$ for a microgel with $0.5 \mathrm{~mol} \%$ BIS is plotted as an example in Fig. 4 . The $T$-dependence of the conductivity is discussed in the plateau region (grey box) where interfacial polarization effects do not play a role. The corresponding conductivity value in this region is defined as DC conductivity $\sigma_{\mathrm{DC}}^{\prime}$ and examined in its temperature dependence.

The samples were heated from $15^{\circ} \mathrm{C}$ to $50^{\circ} \mathrm{C}$ in steps of $1 \mathrm{~K}$ or $2 \mathrm{~K}$ and subsequently cooled down to $15^{\circ} \mathrm{C}$ also in steps. At each temperature an isothermal frequency scan is carried out. This results in an effective heating-cooling rate of approximately $0.2 \mathrm{~K} \mathrm{~min}^{-1}$. For the pNIPAM microgel dispersion a change in the temperature dependence of $\sigma_{\mathrm{DC}}^{\prime}$ is observed (see Fig. 5). Two approximately linear regimes in $\sigma_{\mathrm{DC}}^{\prime}(T)$ can be

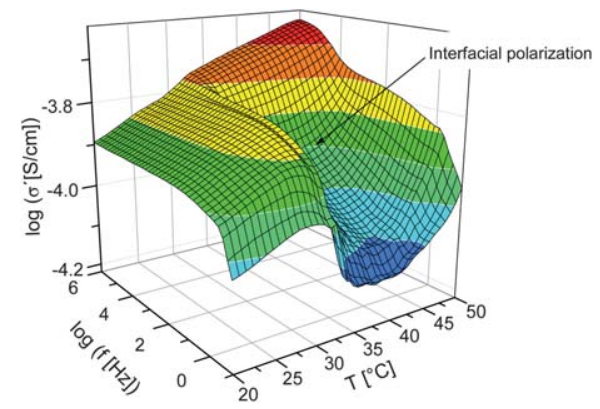

Fig. 3 Real part of the complex conductivity $\sigma^{\prime}$ versus frequency and temperature in a 3D representation for a pNIPAM microgel with a crosslinking density of $0.5 \mathrm{~mol} \% \mathrm{BIS}$. The concentration of the microgel dispersions is $2.5 \times 10^{-2} \mathrm{~g} \mathrm{ml}^{-1}$. 


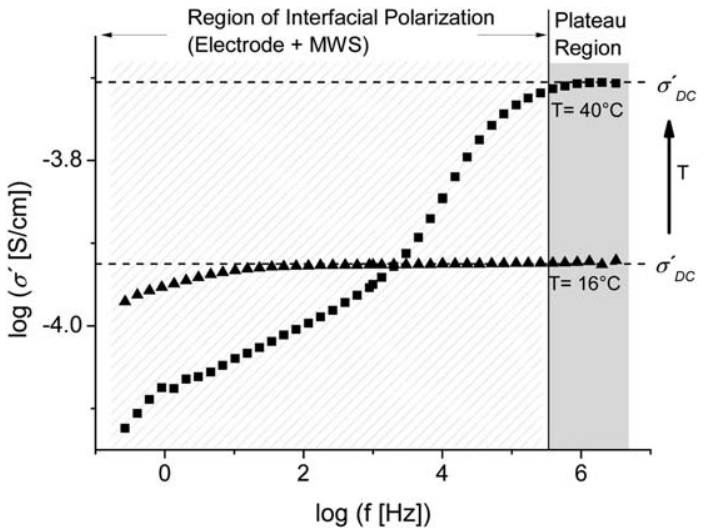

Fig. 4 Frequency dependence of the real part of conductivity $\sigma^{\prime}$ for a microgel with a $0.5 \mathrm{~mol} \%$ crosslinker at $16{ }^{\circ} \mathrm{C}$ (triangles) and $40{ }^{\circ} \mathrm{C}$ (squares). The concentration of the microgel dispersions is $2.5 \times 10^{-2} \mathrm{~g} \mathrm{ml}^{-1}$. The horizontal dotted lines mark the DC conductivity which is obtained in the plateau region.

identified, the one at lower temperatures having a steeper slope than the one at higher temperatures. From the intersection of the two linear fits to the corresponding data a phase transition temperature can be deduced..$^{31,38} \mathrm{~A}$ similar behaviour for all microgels discussed here is observed. It can be explained mainly by two effects, a decrease in effective charge mobility at $T>$ LCST and a reduction in the effective charge number contributing to $\sigma_{\mathrm{DC}}^{\prime}$ at $T>$ LCST. The latter is due to the blocking/entrapping of charge carries at the collapsed particles. These charges do not reach the electrodes and therefore do not contribute to the DC conductivity. This means the effective charge carrier density responsible for DC conductivity is decreased for $T>$ LCST. Moreover because of the present particles the remaining charge carriers have a longer mean diffusion way to reach the electrodes leading to a lower effective mobility (tortuosity effect). ${ }^{31}$ The conductivity generally increases with temperature. Therefore the change in the slope $\mathrm{d} \log \sigma_{\mathrm{DC}}^{\prime} / \mathrm{d} T$ at the phase transition (see Fig. 5) is due to a counterbalance of the temperature effect at the one side and the

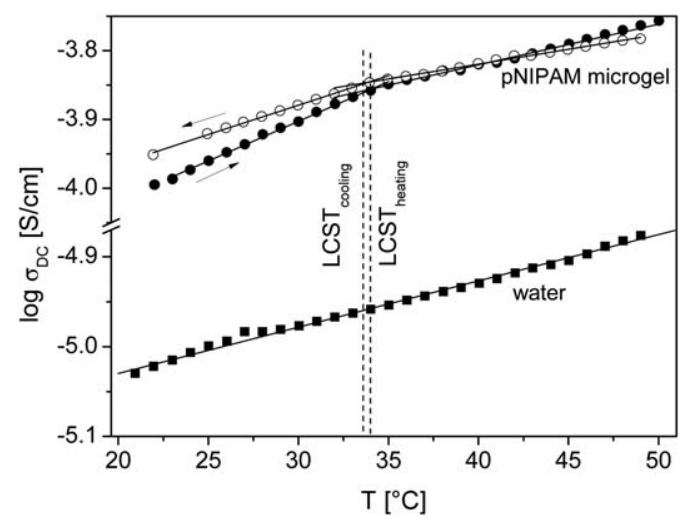

Fig. 5 Temperature dependence of the DC conductivity $\sigma_{D C}^{\prime}$ for Milli-Q ${ }^{\mathrm{TM}}$ water (filled squares) and a pNIPAM microgel with $5 \mathrm{~mol} \% \mathrm{BIS}\left(c=2.5 \times 10^{-2} \mathrm{~g} \mathrm{ml}^{-1}\right.$ in water). Filled circles $=$ heating scan, open circles $=$ cooling scan. Solid lines are linear fits to the corresponding data, dashed lines indicate the change in slope (= LCST) for the microgel dispersion. The arrows in right and left directions mark the heating and cooling scan, respectively. (Please note the break in the $y$-axis). reduced effective charge carrier number with a lower effective mobility at the other side.

The transition temperature was found to be independent of crosslinking density and is located between $34{ }^{\circ} \mathrm{C}$ and $36^{\circ} \mathrm{C}$ for all microgels. The values are slightly higher compared to the LCST deduced from DLS measurements (see Table 1). This is due to different heating-cooling rates employed in DLS and dielectric spectroscopy. For the higher crosslinked microgels (5 mol\% and $10 \mathrm{~mol} \%$ ) the LCST values taken from the heating and cooling scan are in good agreement. For microgels with lower crosslinker content the LCST shows a hysteresis behaviour. This is attributed to kinetic effects for instance the formation of additional hydrogen bonds in the collapsed state between different chain segments when they are overlapped which hinders the swelling process during cooling. ${ }^{39,40}$ With increasing crosslinker content, the chain length between two crosslinking points decreases. Therefore fewer intrachain hydrogen bonds can be formed during the heating scan (collapsing), and the hysteresis becomes smaller and vanishes, respectively. ${ }^{13}$ Similar effects were observed by dynamic light scattering for dilute aqueous solutions of linear pNIPAM,${ }^{41}$ by ultrasensitive differential scanning calorimetry for semidilute pNIPAM solutions ${ }^{42}$ and by dielectric spectroscopy for linear pNIPAM solutions in a wide concentration range. ${ }^{31}$ However, as reported by $\mathrm{us}^{31}$ the hysteresis effect ( $\triangle$ LCST) depends on the heating and cooling rate and vanishes if the rate is around $0.1 \mathrm{~K} \mathrm{~min}^{-1}$. These findings explain the fact that no remarkable hysteresis effect is observed for DLS measurements where the heating-cooling rate is approximately $0.1 \mathrm{~K} \mathrm{~min}^{-1}$.

Moreover, different slopes for heating and cooling are observed below the LCST where the variation of the conductivity with temperature is weaker for the cooling than the heating run. That points out that the deswelling and swelling of the microgel particle take place at different time scales. The same behaviour was observed for linear PNIPAM $^{31}$ and can also be attributed to the additional hydrogen bonds formed in the collapsed state. However one has to differentiate between two effects: the first is the hysteresis in the heating and cooling run, i.e. the $\Delta$ LCST. This effect can be removed by decreasing the heating-cooling rate and giving the system enough time to equilibrate. The second effect is the difference in the slope for heating and cooling below the LCST. Some of the formed hydrogen bonds cannot be completely removed in the cooling process even when the heating-cooling rate is sufficient low. These remaining $\mathrm{H}$-bonds can be considered as new additional crosslinkers ${ }^{13}$ and can only be completely removed at lower temperatures $\left(\sim 4{ }^{\circ} \mathrm{C}\right)$. In such a way the thermal history of the system is "deleted". ${ }^{40}$

\section{Frequency dependence of the complex conductivity $\sigma^{*}(f)$ (interfacial polarization effects)}

The frequency dependence of the complex conductivity $\sigma^{*}(f)$ for microgel dispersions is discussed for temperatures below (swollen particles) and above (collapsed particles) the LCST. All conductivity spectra are normalized with respect to the value of the DC conductivity for the sake of comparison. For the swollen pNIPAM microgel particles the frequency dependence of the 
real part of conductivity $\sigma^{\prime}$ is similar to that of pure water (see Fig. 6). Coming from high frequencies $\sigma^{\prime}$ is independent of frequency over a broad range. This indicates a "normal" conductive behaviour expected for an aqueous solution. ${ }^{25}$ The slight decrease of $\sigma^{\prime}$ with decreasing frequency at low frequencies is due to electrode polarization effects which could not be avoided completely. For temperatures above the LCST where microgel particles are collapsed a pronounced frequency dependence of $\sigma^{\prime}$ is observed. This is demonstrated in Fig. 6 for a microgel with a $0.5 \mathrm{~mol} \%$ crosslinker. These findings are in agreement with the investigations on aqueous solutions of linear pNIPAM. ${ }^{31}$ At temperatures above the LCST the microgels are collapsed and the dispersion can be considered as an inhomogeneous particle suspension. From the dielectric point of view a signature of inhomogeneous systems is the so-called Maxwell-Wagner-Sillars (MWS) polarization ${ }^{25}$ due to the blocking and separation of charge carriers at phase boundaries. Roughly spoken, the separated charges of opposite polarity can be considered as a kind of "dipole" giving rise to an additional contribution to the polarization. The reorientation of this "dipole" due to the charge reversal at the interfaces in the alternating field causes a frequency dependence of $\sigma^{*}(f)$ in addition to the DC conductivity. For colloidal dispersions with spherical particles the frequency dependence of the conductivity due to the MWS polarisation can be modelled by the Hanai equation predictions..$^{43,44}$ For the pNIPAM microgels considered here charges can be blocked or entrapped at the interfaces of the collapsed particles at a mesoscopic length scale. Therefore the strong decrease in the real part of conductivity $\sigma^{\prime}$ with decreasing frequency is assigned to a MWS polarization.

In order to discriminate between the electrode and MWS polarization the real part of permittivity $\varepsilon^{\prime}$ and the imaginary part of conductivity $\sigma^{\prime \prime}$ are analysed for water and a PNIPAM microgel (0.5 mol\% BIS) dispersion (see Fig. 7). Electrode polarization is observed on a macroscopic scale corresponding to the distances of the electrodes at lower frequencies and contributes strongly to the dielectric quantities $\left(\sigma^{*}, \varepsilon^{*}\right)$ in addition to the sample material alone. ${ }^{25}$

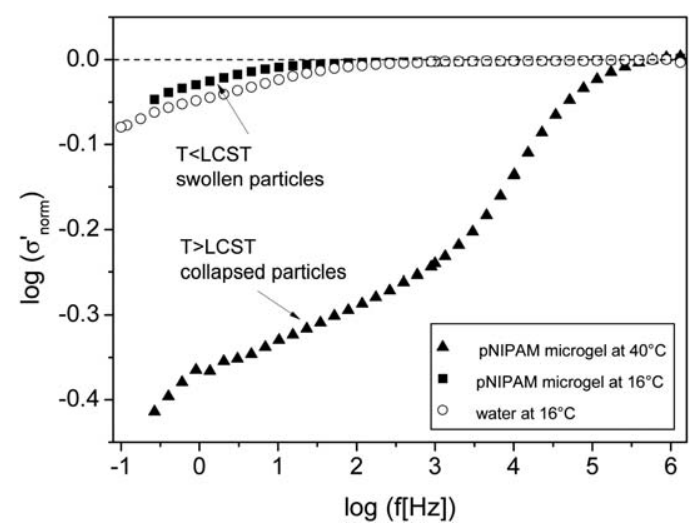

Fig. 6 Frequency dependence of the real part of the normalized conductivity $\sigma_{\text {norm }}^{\prime}$ (curves are normalized to the corresponding value of $\sigma_{\mathrm{DC}}^{\prime}$ ). The concentration of the microgel dispersions is $2.5 \times 10^{-2} \mathrm{~g} \mathrm{ml}^{-1}$.
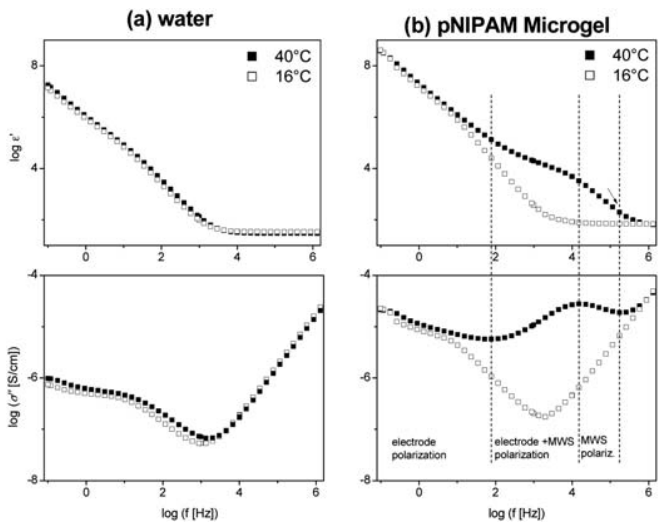

Fig. 7 Real part of the permittivity $\varepsilon^{\prime}$ (top panels) and imaginary part of the conductivity $\sigma^{\prime \prime}$ (bottom panels) versus frequency for water (a) and a pNIPAM microgel $\left(0.5\right.$ mol\% crosslinker, $\left.c=2.5 \times 10^{-2} \mathrm{~g} \mathrm{ml}^{-1}\right)$ (b). The dashed lines indicate the three frequency regions for $T=40^{\circ} \mathrm{C}$ where different processes (as indicated) take place.

For a deepened discussion in addition to the real part of the complex conductivity its imaginary part $\sigma^{\prime \prime}$ as well as the real part of permittivity $\varepsilon^{\prime}$ should also be considered. For pure water only electrode polarization is expected. In that case $\varepsilon^{\prime}$ shows a strong increase for frequencies lower than $10^{3} \mathrm{~Hz}$. In parallel a minimum in the loss part of the conductivity $\sigma^{\prime \prime}$ is observed. This can be considered as the onset of electrode polarization (when going from high to low frequencies). ${ }^{45}$ The behaviour is slightly dependent on temperature. The onset frequency of electrode polarization is located at around $1 \mathrm{kHz}$.

Swollen microgel particles $(T<$ LCST) show a similar dielectric behaviour to water characterized by an onset frequency of electrode polarization of $2 \mathrm{kHz}$. This onset frequency is slightly higher than that for pure water due to a higher conductivity of the microgel dispersion (see Fig. 5). For temperatures above the LCST (collapsed particles) the frequency dependence of $\sigma^{\prime \prime}$ and $\varepsilon^{\prime}$ changes completely and in addition to electrode polarization pronounced effects take place which are assigned to a MWS polarization as discussed above. The minimum in $\sigma^{\prime \prime}$ shown in Fig. 7b gives an onset frequency for the MWS polarization of $173.4 \mathrm{kHz}$. According to the nomenclature developed in ref. 45 it can be considered as fully developed at a frequency of $15.2 \mathrm{kHz}$ which corresponds to the maximum in $\sigma^{\prime \prime}$. Therefore the frequency spectra of the pNIPAM microgel dispersion for $T>$ LCST can be divided into three regions determined by the minima and maximum of $\sigma^{\prime \prime}$ (see Table 2).

The frequency dependence of $\sigma^{\prime}$ for microgels with different crosslinker densities is shown in Fig. 8. For temperatures below

Table 2 Frequency regions with the corresponding polarization effect (plus (+) indicates "yes", minus (-) indicates "no") extracted from Fig. 7 for a pNIPAM microgel with a $0.5 \mathrm{~mol} \%$ crosslinker

\begin{tabular}{llll}
$\begin{array}{l}\text { Polarization } \\
\text { effect }\end{array}$ & $\begin{array}{l}\text { Low } f \text {-region } \\
(\sim 0.1 \text { to } 78 \mathrm{~Hz})\end{array}$ & $\begin{array}{l}\text { Middle } f \text {-region } \\
(\sim 78 \text { to } 15200 \mathrm{~Hz})\end{array}$ & $\begin{array}{l}\text { High } f \text {-region } \\
(\sim 15 \text { to } 174 \mathrm{kHz})\end{array}$ \\
\hline Electrode & + & + & - \\
MWS & - & + & +
\end{tabular}




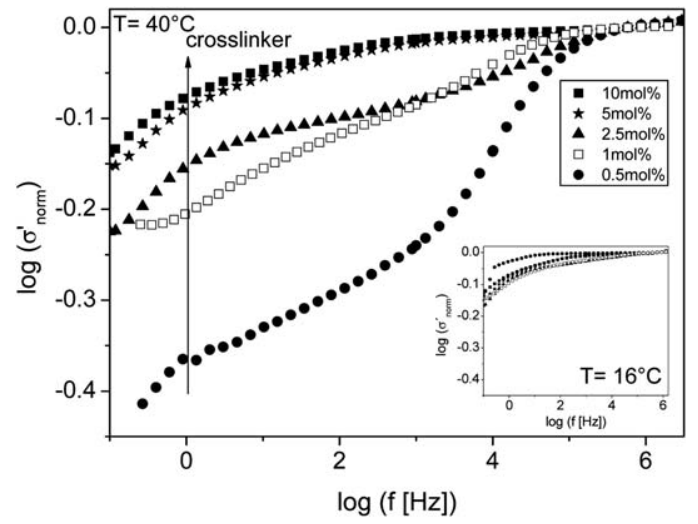

Fig. 8 Frequency dependence of the real part of the normalized conductivity $\sigma^{\prime}$ norm for pNIPAM microgels with different amounts of crosslinker dispersed in water $\left(c=2.5 \times 10^{-2} \mathrm{~g} \mathrm{ml}^{-1}\right)$ at $40^{\circ} \mathrm{C}$ and $16^{\circ} \mathrm{C}$ (inset).

the LCST (see inset Fig. 8) the normalized curves for all concentrations collapse more or less onto a single chart showing only weak frequency dependencies due to electrode polarization. For temperatures above the LCST, MWS polarization effects are observed which are becoming more pronounced with decreasing crosslinker content.

In order to describe the MWS effect in a more quantitative way, the step increase in $\sigma^{\prime}$ is analysed with respect to its height $h$ in dependence on the crosslinker content. The height is defined by taking twice the distance between the point of deflection (position of the maximum of the first derivative of $\log \sigma^{\prime}$ versus $\log f$ ) and the DC conductivity plateau (for more details see ref. 31). The dependence of $h$ on the crosslinker content is shown in Fig. 9. For high crosslinked microgels (10 mol\% BIS) the MWS effect more or less vanishes. As discussed before, pNIPAM microgels can be described by a dense crosslinked core with a shell consisting of lightly connected/branched or linear dangling chains. This results in an inhomogeneous dense core-loose shell

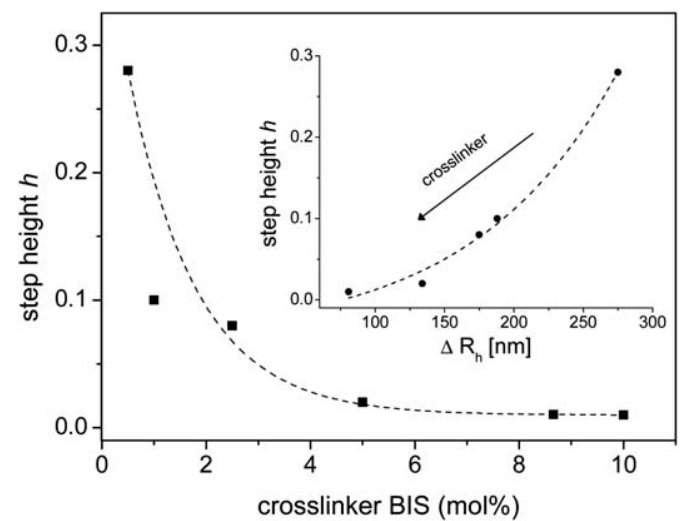

Fig. 9 Step height $h$ measured at $40{ }^{\circ} \mathrm{C}$ in dependence on crosslinker amount. The dotted line shows an exponential fit to the data as a guide for the eyes. The inset shows the correlation between the step height $h$ obtained from dielectric measurements and the change in particle size $\Delta R_{\mathrm{h}}\left(=R_{\mathrm{h}, 20^{\circ} \mathrm{C}}-R_{\mathrm{h}, 45^{\circ} \mathrm{C}}\right)$ extracted from dynamic light scattering experiments for pNIPAM microgels. The crosslinker content increases from right to left. The dotted line is an exponential fit to the data as a guide for the eyes.

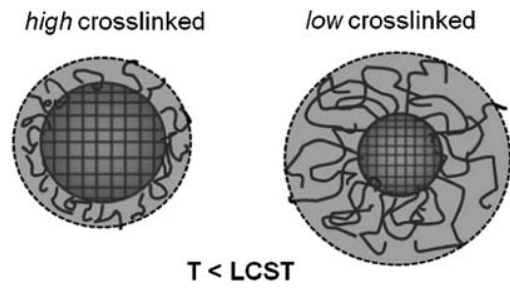

Fig. 10 Simplified scheme (not to scale) of high and low crosslinked microgel particles in the swollen state $(T<$ LCST) in the frame of the structural model discussed in this contribution.

structure with a higher effective number of charges in the shell than in the core. For high crosslinked gels, this structure is less distinctive in the sense that particles are more compact and dense. Low crosslinked gels in turn are looser in structure and exhibit a quite large shell with the characteristics mentioned above. This is schematically illustrated in Fig. 10 for microgel particles below the LCST. However, the core-shell structure has to be regarded as a result of a crosslinker gradient from the inner to the outer part. Upon collapsing of the microgel particles charge carriers are entrapped inside the shell resulting in a pronounced MWS polarization in the case of low crosslinked gels. The fact that high crosslinked gels offer fewer interfaces (within the particle) where ions can be entrapped explains the less pronounced MWS effect.

The relationship between the swelling/deswelling $\operatorname{ratio}\left(\Delta R_{\mathrm{h}}=\right.$ $R_{\mathrm{h}, 20^{\circ} \mathrm{C}}-R_{\mathrm{h}, 45^{\circ} \mathrm{C}}$ ) monitored by dynamic light scattering and the step height $h$ is shown in the inset of Fig. 9. From the foregoing discussion, an increase in $\Delta R_{\mathrm{h}}$ as well as an increase in $h$ can be associated with a decrease in crosslinker density. This correlation between the DLS and dielectric data which are measured completely independent from each other provides strong evidence that the change in the dielectric spectra observed for temperatures above the LCST is directly related to the change in the structure of the microgels taking place at the phase transition.

\section{Conclusion}

The dielectric response of pNIPAM microgel dispersions was discussed in a frequency range of $10^{-1} \mathrm{~Hz}$ to $10^{6} \mathrm{~Hz}$ at temperatures between $15{ }^{\circ} \mathrm{C}$ and $50{ }^{\circ} \mathrm{C}$. Charges were used to probe the internal structure of microgels with different amounts of crosslinker. Therefore the dielectric data were discussed in the conductivity representation $\sigma^{*}(f, T)$. Analogous to solutions of linear pNIPAM $^{31}$ the phase transition could be monitored by the temperature $(T)$ and frequency $(f)$ dependence of conductivity spectra. The LCST determined from the change in slope of the $T$-dependence of the DC conductivity $\sigma_{\mathrm{DC}}^{\prime}$ was found to be independent of the crosslinker amount. Values were slightly higher than the LCST deduced from dynamic light scattering experiments, which is related to the different heating-cooling rates applied in the two measurements.

From the hysteresis observed for cooling and heating in the dielectric experiments one might conclude that the volume phase transition of pNIPAM microgels is probably more of the continuous gel-liquid type. More evidence that the transition is 
continuous comes from time resolved dielectric measurements which will be published elsewhere. ${ }^{46}$ However to clear up this complicated question in more detail additional studies including structural investigations are necessary.

The volume collapse of the microgel particles at the phase transition gives rise to a Maxwell-Wagner-Sillars (MWS) polarization which was observed in the $f$-dependence of the conductivity spectra. The effect was dependent on crosslinking density and more pronounced for lower crosslinked gels. The characteristics of the $T$ - and $f$-dependence of $\sigma^{*}$ were mainly related to the change in the charge mobility, the effective charge number and to charge transport processes including the charge arrangement around the transition point. The effect of crosslinking density on the MWS polarization can be explained by the internal structure of the gels and the unevenly distributed crosslinker density due to the preparation method. Low crosslinked microgels offer more interfaces where charges can be entrapped whereas high crosslinked particles exhibit a denser and more compact structure.

It is shown that the dielectric response of pNIPAM microgels gives information about the phase transition behaviour and the internal structure of such gels. An appropriate model is under development to describe the observed MWS effect more qualitatively and extract characteristic parameters for each of the microgels. The dielectric signatures of the structurally different mircrogels discussed in this contribution can be taken as a starting point for future research and show that dielectric spectroscopy is a suitable method for investigating such thermosensitive systems.

\section{References}

1 M. Das, H. Zhong and E. Kumacheva, Annu. Rev. Mater. Res., 2002, 36, 117-311.

2 M. Karg and T. Hellweg, Curr. Opin. Colloid Interface Sci., 2009, 14, 438-450.

3 S. Nayak, D. Gan, M. Serpe and L. Lyon, Small, 2005, 1, 416-421.

4 F. Zhang and C. Wang, Colloid Polym. Sci., 2008, 286, 889895.

5 L. Zhang, E. S. Daniels, V. L. Dimonie and A. J. Klein, J. Appl. Polym. Sci., 2010, 118, 2502-2511.

6 M. Heskin and J. E. Guillet, J. Macromol. Sci., Chem., 1968, A2, 1441-1455.

7 H. Schild, Prog. Polym. Sci., 1992, 17, 163-249.

8 C. Wu and S. Q. Zhou, Macromolecules, 1997, 30, 574-576.

9 M. Taha, B. S. Gupta, I. Khoiroh and M. J. Lee, Macromolecules, 2011, 44(21), 8575-8589.

10 R. Pelton and P. Chibante, Colloids Surf., 1986, 20, 247-256. 11 W. McPhee, K. C. Tam and R. Pelton, J. Colloid Interface Sci., 1993, 24, 156.

12 R. Acciaro, T. Gilanyi and I. Varga, Langmuir, 2011, 27, 7917-7925.

13 A. Burmistrova, M. Richter, C. Üzüm and R. von Klitzing, Colloid Polym. Sci., 2011, 289, 613-624.

14 K. Kratz, T. Hellweg and W. Eimer, Polymer, 2001, 42, 66316639.

15 I. Varga, T. Gilányi, R. Mészáros, G. Filipcsei and M. Zrínyi, J. Phys. Chem. B, 2001, 105, 9071-9076.
16 T. Tanaka and D. J. Fillmore, J. Chem. Phys., 1979, 70, 1214-1218. 17 M. Stieger, W. Richtering, J. S. Pedersen and P. Lindner, J. Chem. Phys., 2004, 120(13), 6197-6206.

18 N. Dingenouts, S. Seelenmeyer, I. Deike, S. Rosenfeldt, M. Ballauff and P. Lindner, Phys. Chem. Chem. Phys., 2001, 3, 1169-1174.

19 T. Hellweg, K. Kratz, S. Pouget and W. Eimer, Colloids Surf., A, 2002, 202(2-3), 223-232.

20 Y. Matsumura and K. Iwai, J. Colloid Interface Sci., 2006, 296, 102-109.

21 C. D. Jones, J. G. McGrath and L. A. Lyon, J. Phys. Chem. B, 2004, 108, 12652-12657.

22 M. Kuang, D. Wang and H. Möhwald, Adv. Funct. Mater., 2005, 15, 111-1616.

23 T. Hoare and D. McLean, Macromol. Theory Simul., 2006, 15, 619-632.

24 T. Hoare and D. McLean, J. Phys. Chem. B, 2006, 110, 2032720336.

25 Broadband Dielectric Spectroscopy, ed. F. Kremer and A. Schönhals, Springer Verlag, Heidelberg, 2003.

26 Y. Ono and T. Shikata, J. Am. Chem. Soc., 2006, 128, 1003010031.

27 Y. Ono and T. Shikata, J. Phys. Chem. B, 2007, 111, 15111513.

28 S. Nakano, Y. Sato, R. Kita, N. Shinyashiki, S. Yagihara and S. Sudo, J. Phys. Chem. B, 2012, 116(2), 775-781.

29 F. Gómez-Galván, T. Lara-Ceniceros and H. Mercado-Uribe, Meas. Sci. Technol., 2012, 23(2), 025602.

30 J. Zhou, J. Wei, T. Ngai, D. Zhu and J. Shen, Macromolecules, 2012, 45(15), 6158-6167.

31 M. Füllbrandt, R. von Klitzing and A. Schönhals, Soft Matter, 2012, 8, 12116-12123.

32 E. Daly and B. Saunders, Phys. Chem. Chem. Phys., 2000, 2, 3187-3193.

33 A. J. F. Siegert, Tech. Rep., Massachusetts Institute of Technology, Rad. Lab., 1943, vol. 465.

34 S. Provencer, Macromol. Chem., 1979, 180, 201-209.

35 A. Lee, H.-Y. Tsai and M. Z. Yates, Langmuir, 2010, 26(23), 18055-18060.

36 I. Varga, T. Gilanyi, R. Meszaros, G. Filipcsei and M. Zrinyi, J. Phys. Chem. B, 2001, 105, 9071-9076.

37 T. Hellweg, C. Dewhurst, E. Bruckner, K. Kratz and W. Eimer, Colloid Polym. Sci., 2000, 278, 972-978.

38 G. Masci and C. Cametti, J. Phys. Chem. B, 2009, 113, 1142111428.

39 C. Wu, Polymer, 1998, 39, 4609-4619.

$40 \mathrm{H}$. Cheng, L. Shen and C. Wu, Macromolecules, 2006, 39, 2325-2329.

41 C. Wu and X. Wang, Phys. Rev. Lett., 1998, 79, 4092-4094.

42 Y. Ding, X. Ye and G. Zhang, Macromolecules, 2005, 38, 904908.

43 T. Hanai, Kolloid-Z., 1960, 171, 23-31.

44 K. Asami, Prog. Polym. Sci., 2002, 27, 1617-1659.

45 A. Serghei, M. Tress, J. R. Sangoro and F. Kremer, Phys. Rev. B: Condens. Matter Mater. Phys., 2009, 80, 184301.

46 M. Füllbrandt, R. von Klitzing and A. Schönhals, to be published. 